\title{
On large arbitrary girth of a semigroup
}

\author{
Ajmal Ali \\ School of Mathematical Sciences,University of Science,Malaysia ,11800 Penang
}

Received: 5 September 2017, Accepted: 4 November 2017

Published online: 8 January 2018.

\begin{abstract}
This paper is about girth of commuting graph of semigroup. Let $S$ be a finite non-commutative semigroup, its commuting graph, denoted by $G(S)$, is a simple graph (which has no loops and multiple edges) whose sets of vertices are elements of $S$ and whose sets of edges are those elements of $S$ which commute with other elements i.e. for any $a, b \in S$ such that $a b=b a$ for $a \neq b$.

In this paper, we partly solve the the problem posted by J.Araújo, Kinyon M. and Konieczny that for all natural numbers $n \geq 3$ there is a semigroup $S$ such that the girth of $G(S)$ is $n$.
\end{abstract}

Keywords: Commuting graph, girth, semigroup of full transformations.

\section{Introduction}

J.Araújo, Kinyon M. and Konieczny give a construction of band (semigroups of idempotents) in which one can find semigroup of any knit degree $n$, for some positive integer $n$, except $n=3$ [1]. The construction of such type of semigroups also helps for finding semigroups for every $n \geq 2$ such that the diameter of commuting graph $G(S)$ is $n$. On the other hand, finding out the semigroups from a given graph is very important and difficult task in the theory of semigroups. In our paper, we construct semigroup $S$ of large arbitrary even girth for all even natural numbers $n \geq 4$ such that girth of the commuting graph $G(S)$ is $n$.

Suppose that $G(S)$ is commuting graph of some non-commutative semigroup of $S$, then $G(S)=(V, E)$ where $V$ is a finite vertex set and and $E$ is a set of edges such that $E \subseteq\{\{u, v\}: u, v \in V$ for $u \neq v\}$. If $v_{1}, v_{2}, \ldots v_{k}$ are the vertices in $G(S)$ then we write a path $\lambda$ from $v_{1}$ to $v_{k}$ as $\lambda=v_{1}-v_{2}-\ldots v_{k}$ of length $k-1$. A cycle is a path thats starts and ends on the same vertex. The girth of the graph is the length of shortest cycle in the graph.If the graph does not contain any cycle then its grith defined to be infinity.

Let $S$ be a finite non-commutative semigroup whose centre is defined as $Z(S)=\{a \in S: a b=b a \quad \forall \quad b \in S\}$. The commuting graph of a finite non-commutative semigroup is a simple graph whose sets of vertices are from $S-Z(S)$ and whose sets of edges are the elements of $S$ which commute with other elements.i.e for any $a, b \in \mathrm{S}$ such that $a b=b a$ for $a \neq b$. This paper is actually the construction of band (of course non commutative) from given graph such that we can find the even girth of the commuting graph $G(S)$ of semigroup $S$.For the construction of such type of semigroups, our main focus will be on semigroup of full transformations $T(X)$ for a finite set $X$.

Let $T(X)$ be a semigroup of full transformations for a finite set $X$ under the composition of function.Actually the semigroups $T(X)$ is the set of all functions from a finite set $X$ to $X$. In this paper we consider transformations $a, b \in T(X)$ and define composition of functions as $(a b)(x)=a(b(x))$ from right instead of left i.e $(x)(a b)=((x) a) b$ for $x \in X$. 
For $a \in T(X)$ we write image of a by $\operatorname{im}(a)$ and kernel of $a$ is defined as,

$$
\operatorname{ker}(a)=\{(x, y) \in X \times X: a(x)=a(y)\}
$$

and rank of a as $\operatorname{rank}(a)=|\operatorname{im}(a)|$. Also $T(X)$ has $n$ ideals $I_{1}, I_{2} \ldots I_{n}$ where $1 \leq r \leq n$

$$
I_{r}=\{a \in T(X): \operatorname{rank}(a) \leq r\}
$$

Clearly the ideal $I_{1}$ is of rank 1 i.e a constant transformation and hence its commuting graph will be isolated vertices.

\section{Idempotents and zeros elements in semigroups}

In this section, we describe about the notation of idempotent and constant transformations, bands, commuting transformations and left as well as right zero semigroups.

Definition 1. Let $S$ be a semigroup and $e \in S$ is an idempotent if $e^{2}=e$.Also we define the sets of idempotents in $S$ to be $E(S)=\left\{e \in S: e^{2}=e\right\}$ Now, $E(S)$ may be empty or it may be $E(S)=S . I f E(S)=S$ then $S$ is a band. We construct band in our construction in the monoid $T(X)$.

Definition 2. Let $e \in T(X)$ be an idempotent and $\left\{A_{1}, A_{2}, \ldots . A_{k}\right\}$ be a partition of $X$ and unique elements $x_{1} \in A_{1}, x_{2} \in$ $A_{2}, \ldots x_{k} \in A_{k}$ such that for every $i$ we have $A_{i} e=\left\{x_{i}\right\}$. Then the set $\left\{x_{1}, x_{2}, \ldots x_{k}\right\}$ is the image set of e. We use the following notation for $e$,

$$
e=\left(A_{1}, x_{1}\right\rangle\left(A_{2}, x_{2}\right\rangle \ldots\left(A_{k}, x_{k}\right)
$$

If e is a constant transformation with image set $\{x\}$ then we write $(X, x\rangle$ [1].

Definition 3. Let $e=\left(A_{1}, x_{1}\right\rangle\left(A_{2}, x_{2}\right\rangle \ldots\left(A_{k}, x_{k}\right)$ an idempotent in $T(X)$ and let $b \in T(X)$ then $b$ commutes with $e$ if and only if for every $i \in\{1,2 \ldots k\}$ there is a $j \in\{1,2 \ldots k\}$ such that $b x_{i}=x_{j}$ and $b A_{i} \subseteq A_{j}[1]$.

Definition 4. Let e, $f \in I_{r}$ be idempotents and suppose there is $x \in X$ such that $x \in \operatorname{im}(e) \bigcap \operatorname{im}(f)$ then $e-(X, x\rangle-f[1]$.

Definition 5. Let $S$ be a semi group with some binary operation $*$ on it. A zero element is an element $z$ in $S$ such that for all $s$ in $S$, we have, $z * s=s * z=z$. An element in $S$ is called left zero, if all $s$ in $S$, we have, $z * s=z$ and an element is called right zero, if all $s$ in $S$, we have, $s * z=z$.

Definition 6. A semigroup in which every element is a left zero element is called a left zero semigroup.For example, the semigroup $S=\{a, b, c\}$ is a left zero semigroup. Then the Cayley table for $S$ is as given below,

\begin{tabular}{c|ccc}
$*$ & $a$ & $b$ & $c$ \\
\hline$a$ & $a$ & $a$ & $a$ \\
$b$ & $b$ & $b$ & $b$ \\
$c$ & $c$ & $c$ & $c$
\end{tabular}

Definition 7. A semigroup in which every element is a right zero element is called a right zero semigroup.For example, The semi group $S=\{a, b, c\}$ is a right zero semigroup. Then the Cayley table for $S$ is as given below,

\begin{tabular}{c|ccc}
$*$ & $a$ & $b$ & $c$ \\
\hline$a$ & $a$ & $b$ & $c$ \\
$b$ & $a$ & $b$ & $c$ \\
$c$ & $a$ & $b$ & $c$
\end{tabular}


Lemma 1. Let $c_{x}, c_{y}, e \in T(X)$ such that e is an idempotent,then

(1) $c_{x} e=e c_{x}$ if and only if $x \in \operatorname{im}(e)$.

(2) $c_{x} e=c_{y} e$ if and only if $(x, y) \in \operatorname{ker}(e)$.

Proof. (1) Consider $c_{x} e=e c_{x}$. As $c_{x}$ and $e$ commute with each other,therefore,there should be at least one element common in the images of $c_{x}$ and $e$ but $c_{x}$ has only one element in the image set i.e $x$ in $\operatorname{im}\left(c_{x}\right)$. So $x \in \operatorname{im}\left(c_{x}\right) \bigcap \operatorname{im}(e)$ or $x \in\{x\} \bigcap \operatorname{im}(e)$. This implies that $x \in \operatorname{im}(e)$. Conversely, suppose that $x \in \operatorname{im}(e)$.We can write it as $x \in\{x\} \bigcap \operatorname{im}(e)$. This implies that $x \in \operatorname{im}\left(c_{x}\right) \cap i m(e)$. Thus we have $c_{x} e=e c_{x}$.

(2) Consider $c_{x} e=c_{y} e$. As $\operatorname{ker}(e)$ is defined as $\operatorname{ker}(e)=\{(x, y) \in X \times X: x e=y e\}$.Consider $c_{x} e=c_{z}$ and $c_{y} e=c_{t}$ for some $t$ and $z$ in $X$. Thus $c_{z}=c_{t} \Rightarrow z=t$ and hence $z e=t e$. Therefore $(x, y) \in \operatorname{ker}(e)$. Conversely,let $(x, y) \in \operatorname{ker}(e)$ then by def. of $\operatorname{ker}(e)$ we have $x e=y e$, implies $c_{x} e=c_{y} e$.

\section{Construction of the girth of semigroup}

Definition 8. Let $k \geq 2$ be an integer.Let $X=\left\{y_{1}, y_{2}, y_{3} \ldots y_{3 k}\right\}$. We define idempotents $a_{1}, a_{2}, a_{3} \ldots a_{3 k}$ as follows.

For $i \in\{1,2 \ldots 3 k\}$, let $n=\frac{|X|}{3}$, there will be three kernel classes of each $a_{i}$.

class $-1=\left\{y_{1}, y_{2} \ldots y_{n}\right\}$

class $-2=\left\{y_{n+1}, y_{n+2} \ldots y_{2 n}\right\}$

class $-3=\left\{y_{2 n+1}, y_{2 n+2} \ldots y_{3 n}\right\}$

Let $X=A_{1} \bigcup A_{2} \bigcup A_{3}$ be the partition of $X$.Thus we have, $|X|=\left|A_{1}\right|+\left|A_{2}\right|+\left|A_{3}\right|$ such that

class $-1=\left\{y_{1}, y_{2} \ldots y_{n}\right\} \subseteq A_{1}$

class $-2=\left\{y_{n+1}, y_{n+2} \ldots y_{2 n}\right\} \subseteq A_{2}$

class $-3=\left\{y_{2 n+1}, y_{2 n+2} \ldots y_{3 n}\right\} \subseteq A_{3}$

For even n,there will be $\frac{|X|}{3}+1$,odd number of right-zero semigroups generators and even number (2times of odd) of constant generators. For example, for $X=12$ we have $n=4$,so there will be five right-zero semigroups generators and ten (2 times of 5) constant generators whose images sets are defined as;

\begin{tabular}{|l|lll|}
\hline $\operatorname{Im}\left(a_{1}\right)$ & $y_{1}$ & $y_{n+1}$ & $y_{2 n+1}$ \\
\hline $\operatorname{Im}\left(a_{2}\right)$ & $y_{1}$ & $y_{n+2}$ & $y_{2 n+2}$ \\
\hline $\operatorname{Im}\left(a_{3}\right)$ & $y_{2}$ & $y_{n+2}$ & $y_{2 n+3}$ \\
\hline $\operatorname{Im}\left(a_{4}\right)$ & $y_{2}$ & $y_{n+3}$ & $y_{2 n+4}$ \\
\hline $\operatorname{Im}\left(a_{5}\right)$ & $y_{3}$ & $y_{n+3}$ & $y_{2 n+1}$ \\
\hline
\end{tabular}

Table 1: Images of the generators when $n$ is even

For odd n,there will be $\frac{|X|}{3}+1$, even number of right-zero semigroups generators and even number (2times of even) of constant generators. For example,for $X=9$ we have $n=3$, there will be four right-zero semigroups generators and eight (2times of even) whose images sets are defined as;

\begin{tabular}{|l|lll|}
\hline $\operatorname{Im}\left(a_{1}\right)$ & $y_{1}$ & $y_{n+1}$ & $y_{2 n+1}$ \\
\hline $\operatorname{Im}\left(a_{2}\right)$ & $y_{1}$ & $y_{n+2}$ & $y_{2 n+2}$ \\
\hline $\operatorname{Im}\left(a_{3}\right)$ & $y_{2}$ & $y_{n+2}$ & $y_{2 n+3}$ \\
\hline $\operatorname{Im}\left(a_{4}\right)$ & $y_{2}$ & $y_{n+3}$ & $y_{2 n+1}$ \\
\hline
\end{tabular}

Table 2: Images of the generators when $\mathrm{n}$ is odd 
Let $i \in\{1,2 \ldots n\}$ we define the constant transformations as $e_{i}=\left(X, y_{i}\right\rangle=c y_{i}$ i.e all the elements of $X$ maps on the single element $y_{i}$. The semigroup $S$ generated by $S=<a_{1}, e_{1}, a_{2}, e_{2} \ldots a_{n+1}, e_{n+1}>$ is our required semigroup of girth $2\left(\frac{|X|}{3}+1\right)$ i.e 2(no. of generators $\left.a_{i}\right)$.It is easy to see that $Z(S)=\phi$.

Lemma 2. Let $1 \leq i<j$,

(1) $a_{i} a_{i}=a_{i}, a_{j} a_{j}=a_{j}, e_{i} e_{j}=e_{j}, e_{j} e_{i}=e_{i}$

(2) $a_{i} a_{j}=a_{j}, a_{j} a_{i}=a_{i}$

(3) $a_{i} e_{i}=e_{i}, a_{i} e_{j}=e_{j}$

(4) $a_{j} e_{i}=e_{i}, a_{j} e_{j}=e_{j}$

(5) $e_{i} a_{i}=e_{i}, e_{j} a_{i}=e_{j}$

(6) $e_{i} e_{j}=e_{j}, e_{j} e_{i}=e_{i}$

Proof. (1) Proof is obvious by definition 2 that $a_{i}, a_{j}, c_{i}$ and $c_{j}$ are idempotents.

(2) Since the element of the image set of $a_{i}$ properly lies in each $A_{i}$ of $a_{j}$, therefore, $a_{j}$ maps all the elements of $i m\left(a_{i}\right)$ to $\operatorname{im}\left(a_{j}\right)$,thus $a_{i} a_{j}=a_{j}$ forming it right zero semigroup.Similary, $a_{j} a_{i}=a_{i}$. Other proof are similar.

Lemma 3. For each $i, j \in\{1,2 \ldots n\}$,for the adjacent transformations $a_{i}$ and $a_{i+1}$, there will be constant transformation such that $a_{i}-e_{j}-a_{i+1}$ and there will be no path between $a_{i}$ and $a_{i+1}$.

Proof. Since from Lemma 2,we have $a_{i} e_{j}=e_{j}$ and $e_{j} a_{i}=e_{j}$ also $a_{j} e_{j}=e_{j}$ and $e_{j} a_{j}=e_{j}$. This implies $a_{i}-e_{j}-a_{i+1}$.Similarly, $a_{i} a_{j}=a_{j}$ and $a_{j} a_{i}=a_{i}$.This implies there will be no path between $a_{i}$ and $a_{i+1}$.

Lemma 4. For each $i, j \in\{1,2 \ldots n\}$, the cycle $\Pi=a_{i}-e_{j}-a_{i+1}-e_{j}-a_{i+2}-e_{j} \ldots e_{j}-a_{i}$ is unique and minimal cycle in $G(S)$ of length $2 n$.

Proof. Suppose that there is another cycle $\lambda$ in $G(S)$ whose length is $2 n+1$ more that $2 n$ such that $\lambda=a_{i}-e_{j}-a_{i+1}-e_{j}-$ $a_{i+2}-e_{j} \ldots e_{j}-e_{j+1}-a_{i}$. or $\lambda=a_{i}-e_{j}-a_{i+1}-e_{j}-a_{i+2}-e_{j} \ldots e_{j}-a_{i-1}-a_{i}$. We claim that length of $\lambda$ is greater than the length of $\Pi$. Consider the case- 1 when $\lambda=a_{i}-e_{j}-a_{i+1}-e_{j}-a_{i+2}-e_{j} \ldots e_{j}-e_{j}-a_{i}$. From lemma $2, e_{j} e_{j+1}=e_{j+1}$ and $e_{j+1} e_{j}=e_{j}$ thus $e_{j} e_{j+1} \neq e_{j+1} e_{j}$ i.e two constant transformations never commute with each other.Therefore,there will be no path between $e_{j}$ and $e_{j+1}$. Thus there will be no cycle whose length is more than $\Pi$.So $\lambda=\Pi$. Now consider that case-2, $\lambda=a_{i}-e_{j}-a_{i+1}-e_{j}-a_{i+2}-e_{j} \ldots e_{j}-a_{i-1}-a_{i}$. From lemma 3 , there will be no path between $a_{i}$ and ${ }_{i-1}$. Therefore, such type of cycle does not exist.This implies that, $\lambda=\Pi$. So our claim in both cases is wrong.Thus,the cycle $\Pi=a_{i}-e_{j}-a_{i+1}-e_{j}-a_{i+2}-e_{j} \ldots e_{j}-a_{i}$ is unique and minimal cycle in $G(S)$ of length $2 n$.

Now the only case left for the girth of four which is present in the following proposition.

Proposition 1. For all even natural numbers $n \geq 4$, there is a semigroup $S$ of such that girth of $G(S)$ is $n$.

Proof. Let $n=4$.Consider $X=\left\{y_{1}, y_{2}, y_{3}, y_{4}, y_{5}, y_{6}\right\}$. Define the idempotents $a_{1}$ and $a_{2}$ as;

$\operatorname{im}\left(a_{1}\right)=\left\{y_{1}, y_{3}, y_{5}\right\}$ and $\operatorname{im}\left(a_{2}\right)=\left\{y_{1}, y_{3}, y_{6}\right\}$.So there will be three kernel classes of each $a_{i}$ such that,

class $-1=\left\{y_{1}, y_{2}\right\}$

class $-2=\left\{y_{3}, y_{4}\right\}$

class $-3=\left\{y_{5}, y_{6}\right\}$

By lemma 2, $a_{1} a_{1}=a_{1}, a_{2} a_{2}=a_{2}, a_{1} a_{2}=a_{2}, a_{2} a_{1}=a_{1}$ and $a_{1} c y_{1}=c y_{1}, a_{3} c y_{1}=c y_{3}, a_{1} c y_{3}=c y_{3}, a_{2} c y_{3}=c y_{3}$ , $c y_{1} a_{1}=c y_{1}, c y_{1} a_{2}=c y_{1}, c y_{3} a_{1}=c y_{3}, c y_{3} a_{2}=c y_{3}, c y_{1} c y_{3}=c y_{3}, c y_{3} c y_{1}=c y_{1}, c y_{1} c y_{i}=c y_{1}, c y_{3} c y_{3}=c y_{3}$. Thus $S=<a_{1}, e_{1}, a_{2}, e_{3}>$ is the semigroup in which unique and minimal length of cycle $a_{1}-e_{1}-a_{2}-e_{3}-a_{1}$ is 4 . So girth of $S$ is 4 .

Example 1. Let $X=\left\{y_{1}, y_{2}, y_{3}, y_{4}, y_{5}, y_{6}\right\}$. By definition 8, Since $n=\frac{|X|}{3}$ is even so there will be $\frac{|X|}{3}+1$ three right zero semigroups generators namely $a_{1}, a_{2}, a_{3}$ and three constant generators namely $e_{1}, e_{4}, e_{5}$ whose sets of images are defined as; 


\begin{tabular}{|l|lll|}
\hline $\operatorname{Im}\left(a_{1}\right)$ & $y_{1}$ & $y_{3}$ & $y_{5}$ \\
\hline $\operatorname{Im}\left(a_{2}\right)$ & $y_{1}$ & $y_{4}$ & $y_{6}$ \\
\hline $\operatorname{Im}\left(a_{3}\right)$ & $y_{2}$ & $y_{4}$ & $y_{5}$ \\
\hline
\end{tabular}

Table 3: Images of the generators.

Kernel classes of each $a_{1}, a_{2}, a_{3}$ is defined as,

class $-1=\left\{y_{1}, y_{2}\right\}$

class $-2=\left\{y_{3}, y_{4}\right\}$

class $-3=\left\{y_{5}, y_{6}\right\}$

The products defined in lemma 2,we have $S=<a_{1}, e_{1}, a_{2}, e_{4}, a_{3}, e_{5}>$ is the semigroup in which the unique and minimal cycle $a_{1}-e_{1}-a_{2}-e_{4}-a_{3}-e_{5}-a_{1}$ is of length 6 i.e $2\left(\frac{|X|}{3}+1\right)$. Thus girth of $S=6$. The Table 4 presents the Cayley's table for $S$.

\begin{tabular}{l|lllllllll} 
& $a_{1}$ & $a_{2}$ & $a_{3}$ & $e_{1}$ & $e_{4}$ & $e_{5}$ & $e_{2}$ & $e_{3}$ & $e_{6}$ \\
\hline$a_{1}$ & $a_{1}$ & $a_{2}$ & $a_{3}$ & $e_{1}$ & $e_{4}$ & $e_{5}$ & $e_{2}$ & $e_{3}$ & $e_{6}$ \\
$a_{2}$ & $a_{1}$ & $a_{2}$ & $a_{3}$ & $e_{1}$ & $e_{4}$ & $e_{5}$ & $e_{2}$ & $e_{3}$ & $e_{6}$ \\
$a_{3}$ & $a_{1}$ & $a_{2}$ & $a_{3}$ & $e_{1}$ & $e_{4}$ & $e_{5}$ & $e_{2}$ & $e_{3}$ & $e_{6}$ \\
$e_{1}$ & $e_{1}$ & $e_{1}$ & $e_{2}$ & $e_{1}$ & $e_{4}$ & $e_{5}$ & $e_{2}$ & $e_{3}$ & $e_{6}$ \\
$e_{4}$ & $e_{3}$ & $e_{4}$ & $e_{4}$ & $e_{1}$ & $e_{4}$ & $e_{5}$ & $e_{2}$ & $e_{3}$ & $e_{6}$ \\
$e_{5}$ & $e_{5}$ & $e_{6}$ & $e_{5}$ & $e_{1}$ & $e_{4}$ & $e_{5}$ & $e_{2}$ & $e_{3}$ & $e_{6}$ \\
$e_{2}$ & $e_{1}$ & $e_{1}$ & $e_{2}$ & $e_{1}$ & $e_{4}$ & $e_{5}$ & $e_{2}$ & $e_{3}$ & $e_{6}$ \\
$e_{3}$ & $e_{3}$ & $e_{4}$ & $e_{4}$ & $e_{1}$ & $e_{4}$ & $e_{5}$ & $e_{2}$ & $e_{3}$ & $e_{6}$ \\
$e_{6}$ & $e_{5}$ & $e_{6}$ & $e_{5}$ & $e_{1}$ & $e_{4}$ & $e_{5}$ & $e_{2}$ & $e_{3}$ & $e_{6}$
\end{tabular}

Table 4: Cayley table for $S$

Conjecture!. There are no semigroups of a circle and straight line.

\section{Competing interests}

The authors declare that they have no competing interests.

\section{Authors' contributions}

All authors have contributed to all parts of the article. All authors read and approved the final manuscript.

\section{References}

[1] J.Araújo, M.Kinyon and J.Konieczny "Minimal paths in the commuting graphs of semigroups" European J. Combin.,32(1) Pp 178-197, (2011). http://dx.doi.org/10.1016/j.ejc.2010.09.004

[2] J. Araujo and J. Konieczny "Semigroups of transformations preserving an equivalence relation and a cross-section" C. Algebra Vol.32, Pp.1917-1935,(2004).

[3] B.Bundy "The connectivity of commuting graphs" J. Comb. Theory Ser. A 113 ,Pp 995-1007,(2006).

[4] The GAP Group "Algorithms, and Programming" Version 4.4.12,(2008). http://www.gap-system.org

[5] M. Howie "Idempotent generatorsin finite full transformations semigroup" Proc. Roy. Soc. E.Sect A 81(3-4) Pp 317-323.

[6] M. Howie "The sunsemigroup generated by the idempotents of a full transformation semigroup" J London Math soc. 41 Pp 707-716. 
[7] M.Howie "Fundamentals of Semigroup Theory" London Math. Soc. Monogr, Oxford University Press, New York, 1995.

[8] A. Iranmanesh and A. Jafarzadeh "On the commuting graph associated with the symmetric and alternating groups" J. Algebra Appl. 7 , Pp 129-146,(2008).

[9] J. Konieczny "Semigroups of transformations commuting with idempotents" Algebra Colloq. 9 , Pp 121-134,(2002).

[10] L.H. Soicher "The GRAPE package for GAP" Version 4.3, (2006). http://www.maths.qmul.ac.uk/ leonard/grape 